Annuaire suisse de politique de développement

9 | 1990

Annuaire Suisse - Tiers Monde 1990

\title{
II. Politique intérieure et extérieure
}

\section{(2) OpenEdition}

12 Journals

Édition électronique

URL : http://journals.openedition.org/aspd/1301

DOI : 10.4000/aspd.1301

ISSN : 1663-9669

Éditeur

Institut de hautes études internationales et du développement

\section{Édition imprimée}

Date de publication : 1 janvier 1990

Pagination : 69-83

ISSN : 1660-5934

\section{Référence électronique}

«II. Politique intérieure et extérieure », Annuaire suisse de politique de développement [En ligne], 9| 1990, mis en ligne le 28 mars 2013, consulté le 08 septembre 2020. URL : http://journals.openedition.org/ aspd/1301; DOI : https://doi.org/10.4000/aspd.1301 


\section{Politique intérieure et extérieure}

\section{Les débats du Parlement et des commissions sur la coopération au développement}

Au cours de la période couverte par l'Annuaire (de juillet 1988 à juillet 1989), plusieurs débats concernant la coopération au développement à titres divers se sont déroulés au Conseil national et au Conseil des Etats. Nous mentionnons cidessous les thèmes les plus importants abordés au cours des sessions. D'autres motions ou postulats concernant la coopération au développement et l'économie extérieure sont commentés dans les chapitres correspondants.

\section{Crédit-cadre pour l'aide humanitaire}

Les débats parlementaires à propos du nouveau crédit-cadre pour l'aide humanitaire a eu lieu au Conseil national lors de la session d'automne 1988 et au Conseil des Etats en hiver. Le nouveau crédit-cadre s'élève à 530 millions de francs pour trois ans (dès mars 1989) et a été accepté par les deux Chambres. (Voir chapitre "coopération au développement").

\section{Créances de la Confédération dans les pays du Tiers-Monde}

Le Conseil fédéral a répondu en septembre 1988 à une question de Hildbrand (pdc) au sujet des créances de la Confédération dans les pays du Tiers-Monde. Les créances de la Confédération provenant de prêts accordés à des pays du Tiers-Monde se montaient à fin 1987 à 1'595 millions de francs. Le $83 \%$ de cette somme couvre des accords rééchelonnés, $11 \%$ se rapporte à des crédits mixtes et des crédits de transfert et $6 \%$ à l'aide financière. Les taux d'intérêt se situent entre le taux commercial et des taux réduits. 
En 1987, les paiements d'intérêts en provenance des pays en développement s'élevaient à 31 millions de francs et les remboursements à 104 millions de francs.

\section{Facilité d'ajustement structurel du FMI}

Lors de la session de décembre, le Conseil des Etats a traité le prêt de la Confédération de 200 millions de droits de tirage spéciaux (DTS) (cela représente environ $\mathbf{4 0 0}$ millions de francs) pour le programme de facilité d'ajustement structurel renforcée (FASR) du Fonds monétaire international. (cf. le chapitre sur le "fonds monétaire international").

\section{Arrêté sur le sucre}

Par le biais d'une motion issue des milieux de la coopération, le Conseil national a ajouté une clause à l'arrêté sur le sucre, selon laquelle le Conseil fédéral peut autoriser l'importation allant jusqu'à 40'000 tonnes de sucre en provenance des pays en développement à des prix justes dans le cadre de contrats bilatéraux. Un prix plus élevé pour le sucre en provenance des pays en développement que le prix du marché mondial serait le signe de la solidarité des consommateurs et consommatrices d'une Suisse aisée face à ces pays. De plus, ce prix donnerait la possibilité aux pays producteurs de sucre de sortir des monocultures et de diversifier leur agriculture.

Pendant la session de juin 1989, le Conseil des Etats a supprimé la clause de "solidarité" introduite par le Conseil national. Cette mesure serait difficilement applicable et réellement efficace qu'à condition que l'on puisse garantir que ce sont les travailleurs et non les monopoles d'exportation du sucre qui bénéficieraient des augmentations de prix. La conseillère aux Etats Jaggi (ps/ND) a expliqué que ce n'était pas l'importation de matières premières qui devait être encouragée, mais plutôt la capacité des pays en développement de transformer euxmêmes leurs matières premières en y ajoutant une plus-value. D'autres objections concernaient les règles du GATT qui précisent que les contrats bilatéraux ne doivent pas contenir de clauses discriminatoires.

Lors de l'élimination des différences lors de la session de juin 1989, le Conseil national s'est rallié au Conseil des Etats et a voté la suppression de la clause de solidarité avec les pays en voie de développement par 92 voix contre 85 et 5 abstentions.

\section{Politique d'asile}

Dans le cadre de la discussion concernant le rapport d'activités du Conseil fédéral pendant la session de juin 1989, le chef du DFJP, Arnold Koller, a annoncé 
la révision de l'ensemble de la procédure d'asile. II a rejeté la demande des milieux conservateurs de droite en faveur de l'application du droit d'urgence; selon lui, malgré la crise en matière d'asile, ce n'est pas une situation d'exception. En priorité, on examine la possibilité de limiter la procédure de recours ainsi que l'introduction du "séjour provisoire" pour les réfugiés munis d'une décision négative dont le retour dans leur patrie s'avère impossible pour des raisons humanitaires. La procédure d'asile devrait s'accélérer et la durée qui peut actuellement s'élever à trois ans devrait être ramenée à trois ou quatre mois. A la question pourquoi la Suisse ne déposait pas à Strasbourg une plainte contre la Turquie pour violation des droits de l'homme en raison de la situation politique en Turquie et du fort afflux de réfugiés turcs, le conseiller fédéral Felber a répondu qu'une telle plainte était en contradiction avec les principes de la garantie d'asile. Le problème des réfugiés en provenance des pays en voie de développement va marquer les relations internationales ces prochaines années. (Ce thème est traité en détail dans le chapitre "politique d'asile").

\section{Exportation de matériel de guerre}

Une interpellation (Béguin, prd) a fait l'objet lors de la session de juin 1989 d'une prise de position du Conseil fédéral à propos du rôle du Département fédéral des affaires étrangères (DFAE) lors de l'appréciation des demandes d'exportation d'armes. Le parlementaire s'est déclaré étonné par le faible nombre de demandes - 10 au total - soumises au Conseil fédéral entre 1981 et 1987, suspectes pour des raisons humanitaires. Le conseiller fédéral Felber a déclaré que le règlement pour l'exportation du matériel de guerre - en particulier la définition de "matériel de guerre" - allait être examiné par la Commission de gestion du Conseil national.

Le Parlement s'est prononcé en juin sur la situation en Chine. Le Conseil fédéral a annoncé sa décision de stopper ses livraisons de matériel d'armement à la Chine. (Voir chapitre sur les "exportations d'armes" pour plus de détails.)

\section{Stratégie de désendettement}

Lors de la session de juin 1989, le conseiller aux Etats Gadient (udc/GR) a lancé une interpellation au Conseil fédéral demandant ce qu'il comptait entreprendre pour résoudre les graves problèmes d'endettement que connaissent de nombreux pays en développement. Plus précisément, il a demandé si le Conseil fédéral avait l'intention dorénavant d'octroyer plus de crédits à taux d'intérêt réduit en plus de remises de dettes. Selon Gadient, le moment est venu de développer un concept nouveau pour une stratégie globale de désendettement. Dans sa réponse, le conseiller fédéral Stich a indiqué qu'un groupe d'experts travaillait en ce moment sur le problème de l'endettement. 
Convention de l'ONU sur le commerce de la drogue

Dans sa réponse à une question ordinaire de Leuenberger $(p s / \mathrm{ZH})$, le Conseil fédéral a fait savoir en mai 1989 que la Suisse signerait une Convention internationale contre le commerce illégal de la drogue, élaborée dans le cadre de I'ONU. En décembre 1988, un large débat parlementaire avait eu lieu sur le blanchisssage de l'argent sale. (Cf. le dossier sur les "fuites de capitaux").

\section{Relations commerciales entre les banques suisses et l'Afrique du Sud}

En juin 1989, le Conseil fédéral a répondu à une question ordinaire du conseiller national Jean Ziegler (ps/GE) à propos des relations commerciales des banques suisses en Afrique du Sud. II a déclaré en substance que rien n'indiquait que les banques suisses dérogeaient au principe du plafonnement des exportations de capitaux vers l'Afrique du Sud. II n'y avait donc aucune raison de discriminer ces banques et de leur retirer les placements de la Confédération. Le Conseil fédéral a répété que son refus de prendre des sanctions économiques contre l'Afrique du Sud ne pouvait "en aucun cas être interprété comme un soutien à l'apartheid".

\section{Commissions}

La Commission pour les affaires étrangères du Conseil national a discuté également de l'Afrique du Sud lors de sa séance de septembre 1988. Elle juge "gênante" l'augmentation massive des importations de diamants bruts en provenance de l'Afrique du Sud. Depuis 1987, un groupe de travail interdépartemental recense l'ensemble des relations économiques entre la Suisse et l'Afrique du Sud. La Suisse rejette des mesures de boycottage économique à l'encontre de ce pays.

La Commission a approuvé à l'unanimité la poursuite de l'aide humanitaire de la Suisse et a pris connaissance avec satisfaction de l'octroi du crédit de $\mathbf{5 3 0}$ millions de francs pour la période 1989-1992.

La Commission consultative pour la coopération internationale au développement est un organe consultatif du Conseil fédéral en matière de coopération au développement. Sous la direction de la conseillère nationale Blunschy jusqu'à fin 1988, puis du conseiller national Nebiker depuis le début de 1989 , la commission a traité au cours de la période couverte par l'Annuaire (juillet 1988 à juillet 1989) des thèmes suivants:

- méthode de travail et mission de la commission, sujets débattus pour la première fois lors de la séance d'août 1988 et repris en septembre 1989 en présence du conseiller fédéral Felber;

- la coopération technique suisse avec Madagascar ainsi qu'une information 
de l'administration sur les programmes d'ajustement structurel (novembre 1988);

- discussion du projet de message concernant la poursuite de la coopération technique et l'aide financière pour la période 1990 à 1993 (juin 1989);

- discussion du projet de message concernant la poursuite des mesures économiques et commerciales (septembre 1989).

\section{Sources}

Presse et "Bulletin sténographique" des Chambres fédérales

Informations des secrétariats des commissions

Tages-Anzeiger, 3.9.1988; 8.3 et 27.4.1989

NZZ, 5.9, 12.9.1988; 8.3, $12.5,8.6$ et 20.6.1989

\section{La politique d'asile}

La politique suisse d'asile est basée sur la loi sur le droit d'asile, ratifiée en 1979 par les Chambres fédérales et entrée en vigueur au 1er janvier 1981. L'augmentation continue des demandes d'asile et des cas en suspens depuis le début des années quatre-vingt a déjà conduit à deux révisions de cette loi, la première en 1983 et la deuxième en 1987. La procédure de demande d'asile est ainsi accélérée, mais des questions fondamentales concernant le flot toujours croissant des réfugiés en provenance des pays du Tiers-Monde restent sans réponse. Un groupe de travail interdépartemental a publié en janvier 1989 un rapport intitulé: "Stratégie pour la politique des années 90 en matière d'asile et de réfugiés" à l'adresse du Conseil fédéral. Les cantons, les communes, les oeuvres d'entraide et les églises sont appelés à prendre position lors d'une procédure de consultation. Le rapport plaide en faveur d'une politique globale de l'immigration qui intègre la politique d'asile et la politique à l'égard des étrangers.

\subsection{La pratique de l'asile}

Sur la base de la deuxième révision de la loi sur l'asile et de ses ordonnances, les autorités ont appliqué une procédure accélérée, dite procédure 88 (1). Cette procédure accélérée n'a malgré tout pas réussi à diminuer le nombre de cas en suspens dans le délai prévu en raison de la forte augmentation des demandes. En fait, le nombre des requérants à autorisation de séjour provisoire a continué de croître (2) car le renvoi après plusieurs années passées en Suisse n'était, dans la plupart des cas, pas concevable. Le nombre des demandes d'asile a 
Tableau $\mathrm{N}^{\circ} 8$

\section{Statistique sur l'asile 1985-1988}

Demandes et décisions

(Ces chiffres concernent le nombre de personnes et non le nombre de cas)

\begin{tabular}{|c|c|c|c|c|}
\hline & 1988 & 1987 & 1986 & 1985 \\
\hline Demandes d'asile & 16726 & 10913 & 8546 & 9703 \\
\hline $\begin{array}{l}\text { Décisions du DAR, total } \\
\text { décisions positives }{ }^{1} \\
\text { décisions négatives } \\
\text { décisions formelles } \\
\text { (retraits, départs) } \\
\text { Taux d'acceptation } \\
\text { (taux des décisions positives } \\
\text { par rapport au total) }\end{array}$ & $\begin{array}{c}12354 \\
680^{2} \\
8844 \\
2830 \\
\\
5,5 \%\end{array}$ & $\begin{array}{r}11239 \\
829 \\
8292 \\
2118 \\
7,4 \%\end{array}$ & $\begin{array}{r}8879 \\
820 \\
5781 \\
2278 \\
9,2 \%\end{array}$ & $\begin{array}{r}8083 \\
939 \\
5658 \\
1486 \\
\\
11,6 \%\end{array}$ \\
\hline $\begin{array}{l}\text { Cas en suspens } \\
\text { dans les cantons } \\
\text { en première instance } \\
\text { en seconde instance }\end{array}$ & $\begin{array}{r}6218 \\
12648 \\
11197 \\
30063\end{array}$ & $\begin{array}{r}2928 \\
11772 \\
9576 \\
24276\end{array}$ & $\begin{array}{r}2395 \\
12612 \\
6463 \\
21470\end{array}$ & $\begin{array}{r}2129 \\
13258 \\
5533 \\
20920\end{array}$ \\
\hline $\begin{array}{l}\text { Pays de provenance } \\
\text { les plus fréquents }\end{array}$ & $\begin{array}{c}\text { Turquie } \\
9673 \\
\text { Sri Lanka } \\
1516 \\
\text { Yougos- } \\
\text { lavie } \\
818 \\
\text { Inde } \\
730\end{array}$ & $\begin{array}{c}\text { Turquie } \\
5817 \\
\text { Sri Lanka } \\
895 \\
\text { Pakistan } \\
581 \\
\\
\text { Iran } \\
513\end{array}$ & $\begin{array}{c}\text { Turquie } \\
4066 \\
\text { Sri Lanka } \\
951 \\
\text { Sri Lanka } \\
593 \\
\\
\text { Pakistan } \\
392\end{array}$ & $\begin{array}{c}\text { Turquie } \\
3844 \\
\text { Sri Lanka } \\
2764 \\
\text { Zaïre } \\
442 \\
\\
\text { Angola } \\
371\end{array}$ \\
\hline $\begin{array}{l}\text { Acceptation provisoire } \\
\text { Autorisations de séjour } \\
\text { humanitaires }\end{array}$ & $\begin{array}{r}312 \\
2036\end{array}$ & $\begin{array}{l}620^{3} \\
892\end{array}$ & $\begin{array}{l}592^{3} \\
610\end{array}$ & $160^{4}$ \\
\hline
\end{tabular}

1) Asiles accordés dans le cadre d'une action spéciale: 151.

2) Y compris 227 réunions de familles.

3) Jusqu'en 1987: "Internements en régime libre".

4) Internements complets ou partiels.

Source: Statistique du DAR du 25.1.1989, dans: Asyl 1/89 (Ed.: Centrale pour l'aide aux réfugiés) 
augmenté en 1988 de 50\% par rapport à l'année précédente pour atteindre le nombre de $16{ }^{\prime} 726.58 \%$ des requérants provenaient de la Turquie, suivie du Sri Lanka, de la Yougoslavie et de l'Inde. A fin 1988, il y avait encore 30'063 demandes en suspens (en 1987, il y en avait 24'276). Le taux d'acceptation c'est-à-dire le nombre des demandes acceptées par rapport aux décisions matérielles en 1988; ce taux est calculé pour les pays avec au moins 100 décisions matérielles - se situe à $7 \%$, ce qui est plus faible encore que le taux de l'année précédente. II faut relever que ce taux est en constante diminution (il était de $46 \%$ en 1979). Le taux d'acceptation varie fortement selon le pays d'origine: en 1988 , il était de $82 \%$ pour l'Afghanistan, de $0,8 \%$ pour le Zaïre, de $5,3 \%$ pour la Turquie, de 3,7\% pour le Sri Lanka, de 9,3\% pour la Yougoslavie et de 0,5\% pour l'Inde. (Voir tableau "statistique sur l'asile 1985-1988".)

\section{Procédure 88}

La procédure 88 a été introduite en automne de la même année par le Conseil fédéral pour décourager les entrées illégales des requérants d'asile en Suisse. La procédure 88 est appliquée aux réfugiés qui ne sont pas entrés par un des passages frontières officiels. Il est supposé que ces réfugiés illégaux viennent avant tout en Suisse pour trouver du travail. Pour ces requérants, la procédure 88 laisse très peu de chance d'obtenir le droit d'asile. On considère que leur retour ne pose pas de problème. Après un premier interrogatoire par la police cantonale des étrangers (au plus tard quinze jours après l'arrivée en Suisse), ces requérants sont placés dans des centres d'accueil fédéraux en attendant la suite de la procédure. La demande sera traitée en priorité jusqu'à prise de décision. Lors de l'interrogatoire, un représentant d'une organisation d'entraide est présent comme lors de la procédure normale.

De nombreuses organisations et oeuvres d'entraide qui s'occupent de réfugiés critiquent la procédure 88 . L'Oeuvre suisse d'entraide ouvrière (OSEO) demande la suppression de la procédure. A son avis, celle-ci engendre une grande perte de qualité dans le travail de prise de décision et laisse une large place à l'arbitraire.

\section{La Commission de gestion dénonce des irrégularités judiciaires}

La Commission de gestion du Conseil national a reproché au délégué aux réfugiés, Peter Arbenz, d'avoir contrevenu au droit lors des recours et des décisions d'expulsion des deux familles zaïroises Musey et Maza; le délégué aux réfugiés aurait empiété sur les compétences des cantons de Berne et du Jura lors de l'expulsion forcée de la famille Musey en janvier 1988. (La Confédération est responsable de la procédure jusqu'à la prise de décision. L'application de l'expulsion est de la compétence des cantons). Dans le cas Maza, la Commission de gestion est arrivée à la conlusion que les bases légales pour un internement n'existaient pas au départ. 
Après la publication du rapport de la Commission, l'Association de coordination Suisse-Asile, composée de personnes engagées des milieux politiques, culturels, sociaux et religieux, a déposé une pétition en avril 1989 réclamant la suspension du délégué aux réfugiés, Peter Arbenz, et un moratoire contre le refoulement des réfugiés menacés d'expulsion.

\section{Pas de solution globale}

Ces dernières années, de nombreuses voix se sont élevées pour proposer la mise en place d'une solution globale pour les demandes d'asile datant de plusieurs années, ce qui permettrait une diminution des cas en suspens. Lors de la session de mars 1989, le Parlement a refusé une initiative de l'Etat de Fribourg allant dans ce sens, déclarant que la solution globale n'amènerait pas de diminution substantielle car la plupart des anciens cas avaient été traités entretemps.

La commission de pétition a publié en janvier 1989 un petit rapport sur la politique d'asile dans lequel elle propose également une solution globale pour les demandes déposées avant le 1er janvier 1985; elle recommande en outre au Conseil fédéral la suppression du timbrage des passeports avec un $\mathrm{R}$ pour refoulé. Le Parlement a traité ces motions en mars 89 et s'est prononcé à la majorité en faveur de la suppression du timbrage; ce postulat a été transmis.

Le 4 décembre 1988, la majorité du peuple et des cantons a rejeté une nouvelle initiative contre la surpopulation étrangère et pour une réduction du nombre des étrangers en Suisse.

\subsection{Stratégies pour la politique des années 90 en matière d'asile et de réfugiés}

Face aux nombreux problèmes rencontrés dans la pratique de l'asile, des débats politiques et des avis très partagés de l'opinion publique, la Confédération s'est penchée sur l'avenir de la politique d'asile en élaborant un certain nombre de stratégies pour les années 90 . C'est un groupe de travail interdépartemental qui a réalisé ce rapport pour une nouvelle stratégie. II a clairement déclaré que les migrations internationales seraient un des problèmes majeurs de cette prochaine décennie. La politique d'asile sera marquée par les rapports entre les pays du Tiers-Monde et les pays industrialisés.

L'analyse des mouvements migratoires au niveau mondial démontre clairement que l'interdépendance économique croissante et l'augmentation de l'écart entre les pays riches et pauvres accentuent le flux en direction des pays occidentaux à haut niveau de vie. Cette pression est ressentie comme une menace face à laquelle on répond par des mesures restrictives. Celles-ci ont pour con- 
séquence l'augmentation des immigrations illégales et des demandes d'asile. Les refus des pays où la demande a été déposée entraînent une circulation des requérants d'un pays européen à l'autre. Dans le rapport, on estime que le $80 \%$ des refusés restent en Europe, légalement ou illégalement. Selon le rapport, la Suisse reste un des pays phares pour les requérants en raison de sa situation économique favorable et non pas grâce à sa réputation humanitaire.

Les relations de la Suisse avec les différents pays du Tiers-Monde sont marquées par des intérêts très divergents. Les intérêts économiques de la Suisse s'opposent à ceux des pays du Tiers-Monde. La Suisse est en effet un créancier, un importateur de matières premières et un pays protectionniste au niveau de son agriculture. Le groupe de réflexion mentionne dans son rapport que certains problèmes, tel le désendettement, montrent clairement les conflits d'intérêts existants. L'attitude de larges couches de la population est souvent contradictoire face à l'octroi d'autorisations de travail à des requérants d'asile. "Lorsque ceux-ci exercent une activité lucrative, ils sont considérés comme des concurrents. S'ils sont exclus de la vie professionnelle, on les traite de parasites" (rapport p. 56).

La surcharge des autorités chargées des questions d'asile représente un autre problème crucial. Le nombre des demandes et la complexité de la procédure peuvent porter la durée du traitement d'une demande à cinq ans. Cela entraîne alors des problèmes dans le cas d'expulsion après plusieurs années d'attente en Suisse.

Le groupe de réflexion propose les mesures suivantes:

\section{Au niveau de la politique internationale à l'égard des réfugiés:}

le but visé est l'amélioration des conditions politiques, économiques et sociales dans les pays où sévissent la répression politique, la guerre civile ou la guerre ainsi que dans les pays économiquement défavorisés, avec inégalité de répartition des richesses et/ou devant faire face à des crises écologiques.

Les mesures principales sont:

- le désamorcement de la problématique de l'endettement par l'allègement du service de la dette;

- l'encouragement à l'exportation de produits en provenance de pays du Tiers Monde;

- l'intensification de la coopération multilatérale et bilatérale;

- l'harmonisation du droit d'asile entre les différents pays européens.

Au niveau de la politique nationale en matière d'asile:

l'objectif consiste ici à limiter le nombre d'entrées en renforçant les contrôles à la frontière et à lutter contre les filières des passeurs (le 7 octobre 1988, un enfant turc de sept ans est mort d'épuisement lors du passage du col du 
Splugen qu'il passait à pied avec ses parents envoyés illégalement en Suisse par un passeur).

Les propositions les plus importantes sont:

- la limitation des possibilités de travail pour les requérants et la réduction voire l'introduction d'une durée maximum pour les prestations d'assistance;

- l'abaissement de la durée de la procédure à six mois jusqu'à prise de décision définitive grâce à l'augmentation de l'effectif du personnel et à l'introduction d'un système permettant de prendre des décisions collectives;

- la possibilité de demander directement une autorisation de séjour, limitée dans le temps au lieu de déposer une demande d'asile;

- la création d'un droit spécial de procédure d'asile;

- l'étude d'une procédure de recours indépendante de l'administration;

- l'application d'une procédure simplifiée pour les "cas anciens";

- la création d'un office fédéral des réfugiés autonome;

- la mise sur pied de programmes d'aide au rapatriement.

Au niveau de la politique en matière de migrations:

le rapport plaide en faveur d'une politique globale de migrations qui englobe la politique d'asile et la politique à l'égard des étrangers. Jusqu'à maintenant, ces deux domaines ont été strictement séparés et traités de manière totalement indépendante. Mais chaque révision de la loi sur l'asile s'est accompagnée d'une révision de la loi à l'égard des étrangers. Le nombre global d'étrangers résidant en Suisse est déterminant dans l'attitude de la population vis-à-vis des étrangers. Le rapport fait état de la nécessité d'un consensus de base pour la mise en place d'une règlementation globale en matière d'immigration. Le modèle proposé envisage une révision bisannuelle par le Conseil fédéral ou le Parlement concernant les décisions en matière de migrations. II fixerait ainsi le pourcentage d'immigrants autorisés et déciderait de leur composition, vu la grande diversité des catégories d'étrangers. Dans ce modèle, les requérants sont inclus dans la population étrangère résidant temporairement en Suisse. Cela demanderait une révision totale de la loi sur l'asile et de la loi à l'égard des étrangers, voire une modification de la Constitution.

Prises de position sur le rapport en question:

Dans une première prise de position, les différents milieux engagés dans la politique d'asile ont critiqué le rapport du groupe de réflexion, trop unilatéral à leur avis. Le rapport proposerait un durcissement de la politique d'asile et de la politique à l'égard des étrangers. L'Association de coordination Suisse-Asile est d'avis que le groupe de réflexion n'a pas su saisir l'occasion d'intégrer la politique à l'égard des étrangers dans une politique basée sur le respect des droits de l'homme et de la justice sociale dans les pays d'origine des requérants. Le 
Parti socialiste suisse et les syndicats se sont prononcés en principe contre la fusion de la politique d'asile et de la politique en faveur des étrangers en une seule et même politique. Après la fin de la consultation (août 1989), le Conseil fédéral développera une stratégie pour les années 90 en matière de politique d'asile sur la base du rapport et des critiques qu'il a suscitées.

\section{Notes}

1. Les rapports détaillés sur les révisions concernant la loi sur l'asile et la politique en la matière sont publiés dans les annuaires des années $1984,85,86$ et 87/88.

2. L'acceptation provisoire est une mesure de compensation dont la compétence est du domaine du délégué aux réfugiés et qui s'applique aux cas de décision négative où le renvoi n'est pas possible ou ne peut pas être appliqué. Si l'application de la décision s'avère possible plus tard, la mesure de compensation est suspendue et le renvoi est effectif. Voir aussi notes (3) et (4) du tableau de la statistique d'asile 1985-1988.

\section{Sources}

Rapport du groupe de réflexion interdépartemental avec des représentants du DFJP, du DFAE et du DFEP: "Stratégie pour la politique des années 90 en matière d'asile et de réfugiés", Berne, janvier 1989

Département du délégué aux réfugiés

Coordination Suisse-Asile/ Centrale pour les questions d'asile, Berne: pétition pour la suspension du délégué aux réfugiés, Peter Arbenz, 3 mars 1989

NZZ, 15.3., 18/19.3. et 17.5.1989

Tages-Anzeiger, 4.3. et 5.4.1989

Circulaire du MODS 2/89

Asile 1/89, édité par l'Office central suisse d'aide aux réfugiés (OSAR)

\section{Fuites de capitaux}

\section{Lebanon connection}

L'affaire de blanchissage d'argent issu du trafic de drogue, la "Lebanon connection", a provoqué un scandale politique sans précédent, avec notamment la démission de la Conseillère fédérale Elisabeth Kopp (en décembre 1988) et la mise à la retraite anticipée du Procureur général de la Confédération, Rudolf Gerber. Cette affaire de blanchissage a ainsi jeté un éclairage particulier sur les problèmes de fuites de capitaux (voir le dossier dans la partie Analyses et Positions). Le débat n'avait jamais été aussi intense, même pas lors de l'initiative sur les banques de 1984 qui demandait notamment un assouplissement du secret 
bancaire pour décourager l'utilisation de la place financière suisse par la criminalité internationale.

La presse a révélé l'existence en Suisse de réseaux du crime organisé. Elle a aussi relevé l'insuffisance des instruments aux mains de la justice pour lutter contre ces réseaux. Sur la base d'investigations et de rapports de juges suisses ou italiens, la presse a mis en lumière notamment les faits suivants:

- Une gigantesque affaire de blanchissage d'argent provenant du trafic de drogue, par le réseau libano-turc dénommé "Lebanon connection".

- Cette nouvelle affaire de blanchissage d'argent a connu un énorme retentissement surtout par le fait que les frères Magharian sont soupçonnés d'avoir blanchi des narco-dollars par le biais du Crédit suisse et de la société Shakarchi Trading SA. Hans W. Kopp, alors vice-président de cette société, avait été averti par sa femme que le Ministère public de la Confédération enquêtait sur les activités douteuses de la firme en question.

- En fait, le public apprenait peu à peu que grâce aux enquêtes menées sur la Lebanon connection et l'affaire similaire de la Pizza connection, grâce aussi aux enquêtes menées aux Etats-Unis et en Italie, de graves soupçons planaient depuis plusieurs années sur les activités de certaines banques ou sociétés occupées par le commerce des devises (dont la Shakarchi Trading).

- On reconnait maintenant que la loi actuelle ne permet pas de lutter efficacement contre le réinvestissement du capital des organisations criminelles. Celle-ci permet d'arrêter le trafiquant de drogue, la personne qui prépare de l'argent pour l'achat de la drogue, mais non d'arrêter le courrier qui arrive régulièrement en Suisse avec des valises remplies de coupures de banques (ceci grâce à la libre circulation des capitaux). La simple identification des clients prévue par la Convention relative à l'obligation de diligence des banques (révisée en 1987) ne semble pas suffire. Le secteur non bancaire reste encore à l'écart de toute surveillance.

Le Conseil fédéral admet que le secret bancaire rigoureux, la haute qualité des services et la stabilité politique et juridique peuvent favoriser l'attrait que la place financière suisse exerce sur le crime organisé (Message du 12 juin 1989). Pour le Conseil fédéral, il y a blanchissage de fonds lorsque des valeurs patrimoniales d'une organisation criminelle sont systématiquement maquillées au moyen d'opérations financières (change de devises, conversion en monnaie scripturale des espèces...), ceci afin de les soustraire à la perspicacité des organes de poursuite pénale et d'en conserver la valeur économique (Message FF 1989 II, p.965).

Plusieurs procédures ont été engagées ou accélérées suite à l'affaire de la Lebanon connection:

- Dès novembre 1988, enquête de la Commission fédérale des banques auprès des trois grandes banques impliquées dans cette affaire, afin de déterminer leur rôle dans le blanchissage d'argent. 
- Enquête de police judiciaire (dès décembre 1988), puis enquête administrative.

- Acte d'accusation pour violation du secret de fonction contre Mme Kopp, transmis à la Chambre d'accusation du Tribunal fédéral en septembre 1989.

- Mise en place d'une Commission d'enquête parlementaire, menée par le conseiller national Moritz Leuenberger (ps, ZH). Celle-ci a pour mandat d'examiner la gestion du Ministère public et du Département de Justice et Police, de même que la manière d'agir des autorités fédérales et offices fédéraux dans le domaine du recyclage d'argent sale et du trafic de drogue. Le rapport de la Commission a été publié en novembre 1989.

- Accélération de la procédure de révision du code pénal suisse pour réprimer le blanchissage d'argent. Une commission d'étude a préparé une nouvelle norme de décembre 1988 à mars 1989. Le travail reposait sur un projet de disposition pénale élaboré par l'ancien procureur général du Sottoceneri (TI), Me Paolo Bernasconi (voir son article dans la partie Analyses et Positions). Le message du Conseil fédéral a été présenté le 12 juin 1989. Les dispositions prévoient l'emprisonnement ou l'amende pour "celui qui aura commis un acte propre à entraver l'identification de l'origine, la découverte ou la confiscation de valeurs patrimoniales, dont il savait ou devait présumer qu'elles provenaient d'un crime" (article 305bis, Message FF 1989 II,p.979). L'article 305 ter reprend l'obligation de l'identification des clients prévue dans la Convention de diligence des banques, alors que le projet de Paolo Bernasconi allait plus loin, en rendant punissable la négligence des banques. La Commission fédérale des banques soutenait cette proposition, alors que les grandes banques y étaient opposées. Les nouveaux articles du code pénal ont été adoptés sans opposition par le Conseil National le 28 novembre 1989. La proposition de Paul Rechsteiner (ps, SG) pour rendre punissable la négligence des banques et celle de Sergio Salvioni (prd, $\mathrm{TI}$ ) pour inclure la notion d'organisation criminelle ont été rejetées.

\section{Affaire Marcos}

Les avoirs placés dans les banques suisses par l'ex-Président des Philippines Ferdinand Marcos ont été gelés par le Conseil fédéral en mars 1986. Depuis cette date, l'affaire de l'entraide judiciaire avec les Philippines s'enlise dans les méandres des procédures juridiques. Le décès de $F$. Marcos survenu à la fin septembre 1989 ne doit en principe pas empêcher la poursuite des procédures engagées par Manille pour récupérer les fonds détournés. La somme d'argent détournée par les époux Marcos vers la Suisse est évaluée à 1,5 milliards de francs. Certains parlent même d'une somme bien supérieure (plus de 12 milliards). Selon les Philippines, au moins $\mathbf{3 0 0}$ millions de dollars auraient été identifiés et gelés sur des comptes en Suisse. II faut maintenant déterminer si les avoirs appartiennent aux héritiers de Marcos ou à l'Etat philippin. 
En juillet 1987, le Tribunal fédéral jugeait licite de geler ces avoirs. II rejetait ainsi les recours de la famille Marcos.

La procédure d'entraide judiciaire est rendue difficile notamment par le fait que l'argent a été déposé en Suisse soit sous des pseudonymes, soit par le biais d'intermédiaires complices ou de fondations situées en Suisse ou au Liechtenstein. 62 sociétés seraient ainsi impliquées à Zurich, 10 à Genève et 2 à Fribourg. De plus, à chaque étape importante de la procédure; soit la levée du secret bancaire, la transmission de documents à la justice philippine, la restitution des avoirs, des recours peuvent être déposés jusqu'au Tribunal fédéral (Voir aussi l'Annuaire 89, point II,2) .

La levée du secret bancaire devrait permettre aux autorités pénales des cinq cantons concernés (Vaud, Lucerne et les trois mentionnés ci-dessous) d'obtenir les renseignements bancaires nécessaires.

Pour le canton de Zurich, le Tribunal fédéral a rejeté un recours du Crédit Suisse. 30 autres recours émanant de fondations ont été rejetés par le procureur public zurichois en août 1988. Seules trois sociétés étrangères et une fondation du Liechtenstein ont porté un recours auprès du Tribunal fédéral en septembre 1988. Les documents des autres sociétés ont pu ainsi être examinés par la justice. En septembre 1989, les autorités judiciaires ont approuvé la remise des premiers documents bancaires aux enquêteurs philippins. Une trentaine de décisions doivent encore suivre.

En mai 1989, les autorités fédérales ont mis en garde les cantons contre les lenteurs qui marquent la procédure, et qui inquiètent le gouvernement philippin.

A Fribourg, la procédure a été plus rapide. Elle ne concerne pourtant "que" 70 millions de francs, déposés à la SBS. En juin 1988, le canton mettait à disposition de la justice philippine les moyens de preuve et les avoirs. En avril 1989, le Tribunal cantonal rejetait un recours des époux Marcos contre le déblocage de leurs avoirs. Ceux-ci peuvent encore recourir auprès du Tribunal fédéral contre la décision fribourgeoise.

Le juge d'instruction de Genève a accepté la demande d'entraide judiciaire des Philippines en octobre 1988. En mars 1989, l'octroi des documents bancaires (de 4 banques) est accepté (pour des dépôts évalués à 10 millions de francs). Des recours ont aussi été déposés auprès de la Chambre d'accusation cantonale.

Grâce à l'accord bilatéral qui lient les Etats-Unis et la Suisse dans le domaine de l'entraide judiciaire, la procédure peut être plus rapide dans le cas suivant:

En avril 1988, les Etats-Unis demandaient l'entraide judiciaire à la Suisse, dans le cadre d'une procédure pénale américaine contre Ferdinand Marcos. Celui-ci est en effet accusé d'avoir effectué des transactions financières par le biais de la place financière suisse, aux dépens des Etats-Unis et des Philippines. Un versement de l'aide américaine au développement (15 millions de dollars de l'Agence internationale pour le développement) aurait ainsi permis l'acquisition d'immeubles à New-York. Une collection de tableaux avait aussi été volée à l'Etat philippin, avec la complicité du marchand d'armes saoudien 
Adnan Kashoggi, détenu depuis avril 1989 en Suisse, extradé vers les EtatsUnis en juillet 1989, puis libéré sous caution. Les 24 tableaux volés auraient transité par Genève, avant d'aller en France.

Les autorités fédérales ont accepté l'entraide judiciaire, mais un recours a été déposé auprès du Tribunal fédéral. Le 28 juin 1989, celui-ci différait sa décision pour attendre d'avoir les garanties que l'immunité dont bénéficient les chefs d'Etats et leurs proches a bien été retirée officielement aux époux Marcos. Dans le cas contraire, la demande d'entraide ne serait pas valable, car l'immunité exclut la possibilité de procédures pénales d'un pays tiers. Le 12 novembre 89, le Tribunal fédéral a accepté partiellement le recours en refusant l'entraide judiciaire pour l'acquisition d'immeubles à New York.

Haïti: Suite à la demande d'entraide judiciaire haïtienne du 8 avril 1986, l'Office fédéral de la police a bloqué les avoirs de l'ex-Président Jean-Claude Duvalier dans les établissements bancaires de Genève, Lausanne et Zurich (le 15.4. 1986). Le dictateur aurait mis à l'abri des sommes considérables prélevées sur le patrimoine public de son pays, cela en Suisse, en France, en Grande-Bretagne et aux Etats-Unis.

En février 1989, la Chambre d'accusation de Genève a rejeté les recours déposés par les époux Duvalier qui s'opposaient à la remise aux autorités haïtiennes de renseignements et documents saisis dans deux banques genevoises. L'avocat des Duvalier a annoncé un recours auprès du Tribunal fédéral.

\section{Sources}

Message du Conseil fédéral concernant la modification du code pénal suisse (Législation sur le blanchissage d'argent et le défaut de vigilance en matière d'opérations financières), du 12 juin 1989. FF II 1989, 961

Tages-Anzeiger, $4.11,5.11,8.11,10.11$ et $12.11 .1988 ; 19.1,1.2,14.3,12.4,12.7,11.9$, 22.9 et $29.9,3.11 .1989$

NZZ, 15.12 et 16.12.1988; $20.1,1.2,16.2,13.3,11.5,30.5,10 / 11.6,23.6,29.6$ et 29.9.1989

Le Courrier, 25.10 et $28.11 .1988 ; 12.1,20.1,16.2,28.4,29.6,24.8$ et 29.9.1989

Journal de Genève, 29.6.1989

L'Hebdo, 15.12.1988; 26.1, 9.2 et 11.5.1989 